\title{
JGraph - A Java Based System for Drawing Graphs and Running Graph Algorithms
}

\author{
Jay Bagga ${ }^{1}$ and Adrian Heinz ${ }^{2}$ \\ 1 Ball State University, Muncie IN 47306, USA \\ ${ }^{2}$ Ontario Systems, Muncie, IN 47304, USA
}

\section{Introduction}

JGraph is a Java based system for drawing graphs and for running graph algorithms. A number of well-known algorithms are provided, including those for planarity testing and drawing planar graphs on a grid. The algorithms can be run with an animation feature where the user can see the intermediate steps as the algorithm executes. The system is extensible in that new algorithms can be easily added.

The JGraph project was conceived and led by Jay Bagga. Several of his graduate students have made contributions. Aaron Nall designed the original version and the graphical user interface. The most recent version has been managed by Adrian Heinz who has added many new features and has also implemented algorithms for planarity testing and drawing of planar graphs on a grid.

This system was initially created for students in computer science, mathematics, and other related disciplines who are learning graph theory and using graph algorithms. It can be used by anyone with an interest in graph drawing and graph algorithms.

To draw a planar graph on a grid, we use the Shift Method of de Fraysseix et. al. 12

\section{Architecture}

\subsection{Programming Language}

The source code is written in Java, and we have used Java Swing components.

\subsection{Operating System}

The code can be compiled to run on multiple platforms. We have been running it on PCs with MS-WINDOWS 95/98/2000 and JDK 1.3 installed.

P. Mutzel, M. Jünger, and S. Leipert (Eds.): GD 2001, LNCS 2265, pp. 459-460, 2002. (C) Springer-Verlag Berlin Heidelberg 2002 


\section{$3 \quad$ Features}

The graphical user interface is designed so that a user can draw graphs on the screen almost as one would on paper. The vertices and edges are drawn by clicking and dragging the mouse. Properties such as colors of vertices and edges, labels, and weights can be modified. Vertices and edges can be easily moved to any position. A number of example graphs are provided. Some graph operations such as rotation are also provided. Many well-known graph algorithms are available. These include depth-first, breadth-first search, minimum spanning tree, shortest path, blocks-finding, and planarity-testing. Also included is an implementation of an algorithm for drawings of planar graphs on a grid.

New algorithms can be easily added to the system. Algorithms can be selected from a menu. Running the algorithm in a new window preserves the original graph. If the animated running algorithm option is selected, the intermediate steps are shown in the window as the algorithm runs.

The figure below shows a maximal planar graph drawn on a grid by the JGraph software.

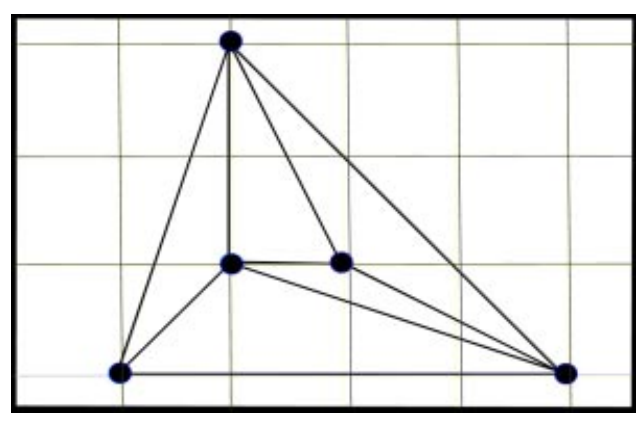

\section{Conclusion}

The JGraph system has been used as a learning tool. A version of this system is available on the web (http://www.cs.bsu.edu/homepages/gnet.) This is an ongoing project. New features and algorithms will continue to be added. Suggestions and comments are greatly welcome. The first author can be contacted at jbagga@bsu.edu.

\section{References}

1. H. de Fraysseix, J. Pach and R. Pollack, "How to draw a planar graph on a grid," Combinatorica 10(41-51), 1990.

2. Shin-ichi Nakano, "Planar Drawings of Plane Graphs," IEICE Trans. Fundamentals E00-A(1-9), 2000. 